\title{
Neuroprotective Effect of Phosphocreatine on Focal Cerebral Ischemia-Reperfusion Injury
}

\author{
Tiegang Li, ${ }^{1}$ Nana Wang, ${ }^{2}$ and Min Zhao ${ }^{1}$ \\ ${ }^{1}$ Emergency Department, Shengjing Hospital of China Medical University, Shenyang 110004, China \\ ${ }^{2}$ Endocrinology Department, Shengjing Hospital of China Medical University, Shenyang 110004, China
}

Correspondence should be addressed to Min Zhao, zhaom@sj-hospital.org

Received 5 November 2011; Revised 3 January 2012; Accepted 3 January 2012

Academic Editor: George Perry

Copyright ( $) 2012$ Tiegang Li et al. This is an open access article distributed under the Creative Commons Attribution License, which permits unrestricted use, distribution, and reproduction in any medium, provided the original work is properly cited.

\begin{abstract}
Phosphocreatine (PCr) is a natural compound, which can donate high-energy phosphate group to ADP to synthesize ATP, even in the absence of oxygen and glucose. At present, it is widely used in cardiac and renal ischemia-reperfusion (IR) disease. In this study, to examine the protective efficacy of PCr against cerebral IR, disodium creatine phosphate was injected intravenously into rats before focal cerebral IR. Intracranial pressure (ICP), neurological score, cerebral infarction volume, and apoptotic neurons were observed. Expression of caspase-3 and aquaporin-4 (AQP4) was analyzed. Compared with IR group, rats pretreated with PCr had better neurologic score, less infarction volume, fewer ultrastructural histopathologic changes, reduced apoptosis, and lower aquaporin-4 level. In conclusion, PCr is neuroprotective after transient focal cerebral IR injury. Such a protection might be associated with apoptosis regulating proteins.
\end{abstract}

\section{Introduction}

Transient cerebral ischemia-reperfusion (IR) injury is a major complication in stroke, resuscitation, and perioperative period, in which $50-70 \%$ survivals suffer from severe disabilities [1]. Decrease of ATP after anoxia or ischemia has been shown to be one of the original steps to cause the following injuries, and the creatine/phosphocreatine system can to some extent compensate for the injuries of anoxia or ischemia. In normal condition, creatine $(\mathrm{Cr})$ is synthesized in the brain and is used to form phosphocreatine (PCr) with a reaction catalyzed by creatine kinase (CK), called Lohmann reaction. When the phosphate group is detached from ${ }^{1} \mathrm{PCr}$, $45 \mathrm{~kJ} / \mathrm{mol}$ of free energy become available compared with only 31.8 for ATP [2]. Thus, PCr can donate its phosphate group to ADP to resynthesize ATP under conditions of ATP exhaustion. In this way, PCr allows ATP synthesis even in the absence of oxygen and glucose $[3,4]$

$$
{ }^{1} \mathrm{Cr}+\mathrm{ATP} \stackrel{\mathrm{CK}}{\longleftrightarrow} \mathrm{PCr}+\mathrm{ADP}+\mathrm{H}^{+} .
$$

Some studies showed that $\mathrm{PCr}$ could improve the outcome after neonatal hypoxic ischemic encephalopathy [5]. To investigate whether $\mathrm{PCr}$ has protective effect on focal cerebral IR injuries, we introduce exogenous PCr to rats before cerebral infarction and studied the effect of $\mathrm{PCr}$ on intracranial pressure (ICP), neural dysfunction score, cerebral infarction volume, cell apoptosis, expression of caspase-3, and level of aquaporin-4 (AQP4) in brain.

\section{Materials and Methods}

2.1. Rats. SPF grade Wistar rats, weighing $250-350 \mathrm{~g}$, were obtained from Animal Center, Shengjing Hospital of China Medical University. The rats were housed in SPF facilities with a $12 \mathrm{~h}$ dark-light cycle in Animal Center of Shengjing Hospital of China Medical University and fed ad libitum in experiment. The animal use protocols used were approved by the Institutional Animal Care and Use Committee at China Medical University. All animal experiments were carried out in accordance with the National Research Council Guide for the Care and Use of Laboratory Animals, as adopted by the National Institutes of Health.

2.2. Method of Animal Model. Focal cerebral IR [6] was achieved using transient middle cerebral artery occlusion 
(MCAO). Anesthesia was induced by $10 \%$ chloral hydrate $(0.3 \mathrm{mg} / \mathrm{kg}$ i.p.). The left common carotid artery (CCA), external carotid artery (ECA), and internal carotid artery (ICA) were identified, and the three were ligated near bifurcation. After careful preparation of the vessel, a small incision was made on the common carotid artery and a $0.2 \mathrm{~mm}$ monofilament nylon suture was introduced through the common carotid artery into the internal carotid artery (about 18 to $20 \mathrm{~mm}$ from the external-internal carotid artery bifurcation). At the end of a 2-hour ischemic period, blood flow was restored by carefully removing the nylon filament. During surgery, rats were maintained at constant temperature using a heating pad.

According to Zea Longa score standard [7], the model was qualified when it scored 2-4 (score 0 , normal, no neurological sign; score 1 , cannot completely stretch contralateral forelimbs; score 2, contralateral circling when walking; score 3 , contralateral fall over when walking; score 4, cannot walk, and lowering of consciousness.).

\subsection{Animal Treatment}

2.3.1. S Group (The Sham-Operated Group). The left common carotid artery (CCA), external carotid artery (ECA), and internal carotid artery (ICA) were exposed but not ligated in the operation.

2.3.2. IR Group. The rats were operated by the method of animal model, at the end of a 2-hour ischemic period, blood flow was restored by carefully removing the nylon filament.

2.3.3. P1 Group. $100 \mathrm{mg} / \mathrm{kg}$ of disodium creatine phosphate (Alfa Wassermann, Inc., disodium creatine phosphate was dissolved in saline in the concentration of $50 \mathrm{mg} / \mathrm{mL}$ ) was administered intravenously $30 \mathrm{~min}$ before operation.

2.3.4. P2 Group. These rats received the same IR, but, in contrast to the IR group, $200 \mathrm{mg} / \mathrm{kg}$ of disodium creatine phosphate was administered intravenously $30 \mathrm{~min}$ before operation.

2.3.5. P3 Group. These rats received the same IR, but, in contrast to the IR group, $400 \mathrm{mg} / \mathrm{kg}$ of disodium creatine phosphate was administered intravenously $30 \mathrm{~min}$ before operation.

We observed neurological deficits at $24 \mathrm{~h}$ and $72 \mathrm{~h}$ after occlusion, and intracranial pressure (ICP) was determined at $1 \mathrm{~h}, 2 \mathrm{~h}, 4 \mathrm{~h}$, and $24 \mathrm{~h}$. Apoptosis detection by TUNEL and caspase- 3 immunohistochemical staining, aquaporin- 4 (AQP4), and ultrastructure observation were detected at $24 \mathrm{~h}$ and $72 \mathrm{~h}$ after ischemia.

2.4. Neurological Score. $24 \mathrm{~h}$ and $72 \mathrm{~h}$ after the onset of ischemia, neurologic evaluation was performed by the same investigator, who was not aware of the group assignment. Motor behavior was evaluated using 4 tests in each rat before sacrificed [8]. The specific tests included (a) observation of spontaneous ipsilateral circling, graded from 0 (no circling) to 3 (continuous circling); (b) contralateral hindlimb retraction, which measured the ability of the animal to replace the hindlimb after it was displaced laterally by 2 to $3 \mathrm{~cm}$, graded from 0 (immediate replacement) to 3 (replacement after minutes or no replacement); (c) beam walking ability, graded 0 for a rat that readily traverses a $2.4 \mathrm{~cm}$ wide, $80 \mathrm{~cm}$ long beam to 3 for a rat unable to stay on the beam for 10 seconds; (d) bilateral forepaw grasp, which measures the ability to hold onto a $2 \mathrm{~mm}$ diameter steel rod, graded 0 for a rat with normal forepaw grasping behavior to 3 for a rat unable to grasp with the forepaws.

2.5. Intracranial Pressure. ICP [9] was monitored continuously from the cisterna magna at $50 \mathrm{~Hz}$ using AcqKnowledge software (v3.7.3, Biopac, Systems, Santa Barbara, CA). A dorsal midline occipitocervical incision was made, and the muscles were dissected off the occiput, atlas and dura overlying the cisterna magna. A 27-gauge needle, attached using short, noncompliant tubing to a pressure transducer (TSD104A; Biopac Systems, Santa Barbara, CA), was inserted into the cisterna magna. Reported ICP values are averaged over 25 min.

\subsection{Cell Apoptosis Detection}

2.6.1. TUNEL Assay. Brain was sampled after reperfused with sodium chloride and $4 \%$ formaldehyde, regularly embedded in paraffinum, and then sectioned at a thickness of $4 \mu \mathrm{m}$. The level of cell apoptosis was determined with a Roche in situ cell-apoptosis-assay kit with nuclei stained in brown particles (Roche Diagnostics, Indianapolis, IN). 5 high-power fields $(\times 400)$ were randomly selected, and the number of apoptotic cells was counted for each field. Apoptosis index $(\mathrm{AI})=$ number of positive cells/number of total cells.

2.6.2. Caspase-3 Immunohistochemical Assay. Caspase-3 immunohistochemical procedures were performed strictly according to the manufacturer's guidelines (Nanjing Jiancheng Bio Co., Ltd). And the slides with the addition of $0.01 \mathrm{mmol} / \mathrm{L}$ PBS (containing $1: 200$ nonimmunity animal serum), instead of primary antibody, showed no response. Under the microscope, those with brown granules in cytoplasm were considered as positive cells and were counted in 5 random high-power fields $(400 \times)$.

2.7. Electron Microscope Procedure. Materials of $1 \mathrm{~mm}$ thickness were fixed for 2 hours in 4\% glutaraldehyde (pH 7.2-7.4, buffered with phosphate) and postfixed with $1 \%$ osmium tetroxide in phosphate buffer ( $\mathrm{pH} 7.4)$ for 2 hours at $4^{\circ} \mathrm{C}$. Dehydration was carried out with ethyl alcohol in a $50 \%$ concentration up to absolute. The materials were embedded in Epon resin. Ultrathin sections were cut, stained with uranyl acetate and lead citrate. Specimens were observed and photographs taken using an electron microscope. The evaluator of the tissue was blinded to the study objective and the intervention . 
2.8. Western Blot of AQP4. The protein level of AQP4 [9] was determined by Western Blot analysis using a rabbit monoclonal antibody against rat AQP4 (Santa Cruz). Brain samples were pulverized, incubated in lysis buffer and protease inhibitor cocktail (Sigma) for $1 \mathrm{~h}$ at $4^{\circ} \mathrm{C}$. After a $10 \mathrm{~min}$ centrifugation at 12000r, the supernatant was collected, and the protein content was quantified. $30 \mathrm{mg}$ protein was loaded per lane, separated by $12 \%$ SDS-PAGE electrophoresis, and electroblotted onto nitrocellulose membranes for $1.5 \mathrm{~h}$ at a constant voltage of $10 \mathrm{~V}$ using semidry apparatus. The membrane was incubated with a rabbit monoclonal anti-rat AQP4 antibody (Lab Vision, Fremont, CA) overnight at $4{ }^{\circ} \mathrm{C}$ and then incubated with an anti-rabbit immunoglobulin $G$ horseradish peroxidase-conjugated secondary antibody (Pierce, Rockford, IL). The signals were detected with an enhanced chemiluminescence kit (Pierce) and exposed on $\mathrm{x}$-ray film. After the film was scanned with a GS-700 imaging densitometer (Bio-Rad, Hercules, CA), a quantitative analysis was performed using Multi-Analyst software (Bio-Rad).

2.9. Statistics. All data were presented as mean $\pm S D$ and analyzed by SPSS 16.0 software. Data of ICP, AI, caspase-3 positive cells, and level of AQP4 were employed by repeatedmeausures ANOVA for multiple comparisons. Neurological score was compared by Kruskal-Wallis test. If the result was significant, ranks of arranged score were analyzed by Student-Newman-Keuls test for multiple comparisons. $P<0.05$ was regarded as significance.

\section{Results}

High-dose usage of disodium creatine phosphate can cause low blood pressure. In the P3 group, 5 rats were dead from unconsciousness, which was thought to be related to low blood, pressure. 2 rats from the IR group, 1 from the P2 group and 1 from the $\mathrm{P} 3$ group were excluded from this study because of the development of subarachnoid hemorrhage. 1 rat from the IR group, 1 rat from the P2 group, 2 rats from the P3 group were excluded from this study because they were not scored 2-4 Zea Longa score.

3.1. Neurological Score. $24 \mathrm{~h}$ and $72 \mathrm{~h}$ after the onset of ischemia, neurologic evaluation was evaluated. There was no neurological deficit in the sham-operated group. Neurological score after ischemia reperfusion is shown in Table 1. There was significant alleviation in motor function in $\mathrm{PCr}$ pretreated group than that in the IR group.

3.2. Intracranial Pressure. The ICP of the five groups was shown in Figure 3. ICP was lower in the IR, P1, P2, P3 groups than that in the sham-operated group during ischemia stage. But ICP was higher in the IR and PCr-pretreated group when compared with the sham-operated group after reperfusion. But there were no statistically significant differences among the five groups at all the time points (Figure 1).

3.3. Cell Apoptosis. TUNEL and caspase-3 immunohistochemical assay were adopted to determine neuronal apop-
TABLE 1: Neurologic score after 24 and $72 \mathrm{~h}$ of ischemia after middle cerebral artery occlusion.

\begin{tabular}{|c|c|c|}
\hline \multirow{2}{*}{ Group } & \multicolumn{2}{|c|}{ Neurological score } \\
\hline & $24 \mathrm{~h}$ & $72 \mathrm{~h}$ \\
\hline IR group & $7.31 \pm 2.03$ & $5.63 \pm 1.41$ \\
\hline P1 group & $6.36 \pm 1.36^{*}$ & $4.84 \pm 1.62^{*}$ \\
\hline P2 group & $5.11 \pm 1.22^{*, \dagger}$ & $2.61 \pm 1.63^{*, \dagger}$ \\
\hline P3 group & $4.95 \pm 1.43^{*, \dagger, \ddagger}$ & $1.95 \pm 1.16^{*, \dagger}$ \\
\hline \multicolumn{3}{|c|}{$\begin{array}{l}{ }^{*} P<0.01 \text {, versus IR group. There were significant differences of } \\
\text { neurological score in P1, P2, P3 groups than that in IR group; }{ }^{\dagger} P<0.05 \text {, } \\
\text { versus P1 group. There were significant differences of neurological score } \\
\text { in P2, P3 groups than that in P1 group; }{ }^{\ddagger} \text { versus P2 group. There were } \\
\text { significant differences of neurological score between P2 and P3 groups. But } \\
\text { in } 72 \mathrm{~h} \text {, there was no significant difference of neurological score between P2 } \\
\text { and P3 groups. }\end{array}$} \\
\hline
\end{tabular}

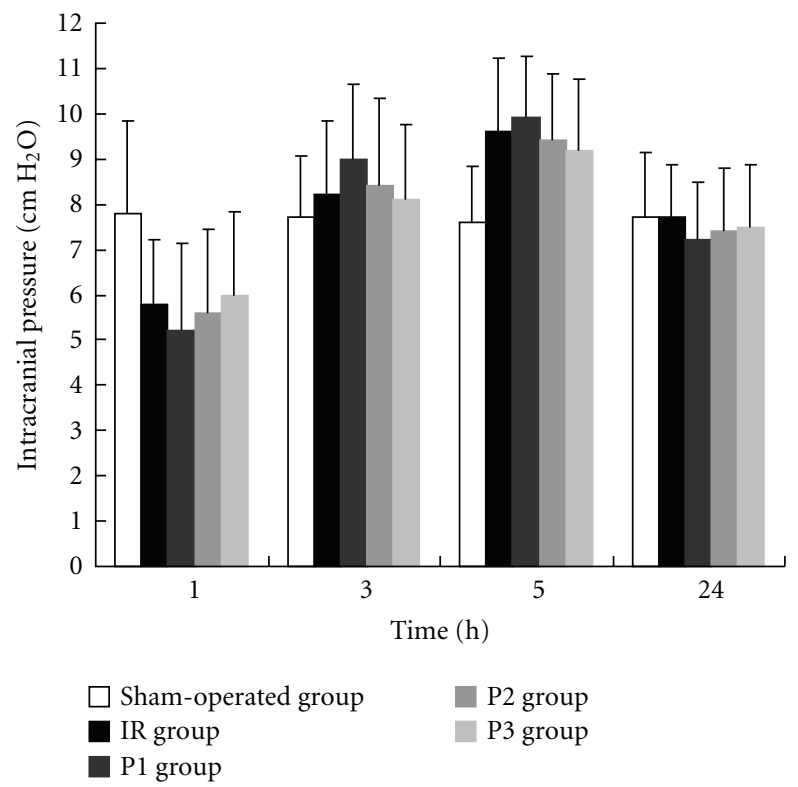

Figure 1: Intracranial pressure of five groups. There were no obviously differences of ICP among five groups in serial time points.

tosis. In the study, the apoptosis cells were mainly on the fridges of ischemia area. AI value and caspase- 3 positive cells were significantly decreased after PCr treatment (Figures 2, 3 , and 4 ).

3.4. Ultrastructure. Clear bilayer nuclear membrane and nucleolus, rounded or oval-shaped mitochondria, were seen in neuron cells in sham-operated group. In the IR group, shrunken neucleus and aggregated chromatin toward the nuclear membrane were observed. In obviously swollen mitochondrion, cristae were disordered, cracked, or decreased, and membrane structure became incomplete or partially disappeared. In the P1 group, the nuclear membranes were clear, and the granule-like aggregation of chromatin could be seen. But organelles were also disordered. In the P2 group, slightly pycnosis, complete nucleus membrane, and less degenerated organelles could be observed. In the P3 group, 


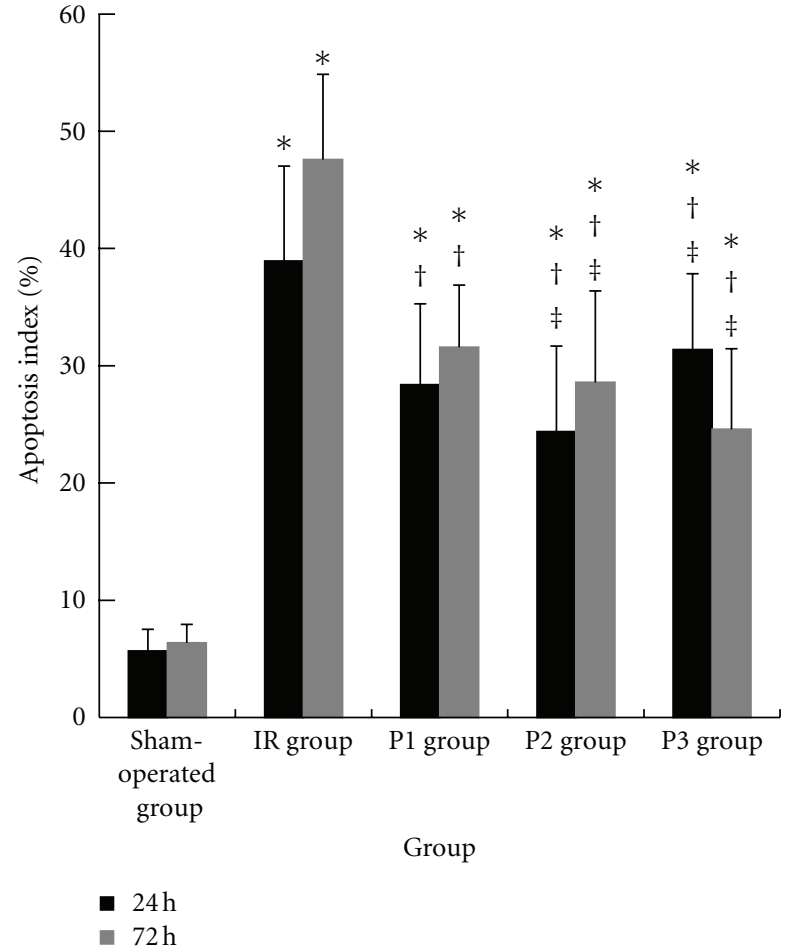

FIGURE 2: Apoptosis index in brain slices of five groups at $24 \mathrm{~h}$ and $72 \mathrm{~h}$. Data are presented as means $\pm \mathrm{SD}$. ${ }^{*} P<0.01$, versus shamoperated group. There were significant differences of apoptosis index in IR, P1, P2, P3 groups than that in sham-operated group; ${ }^{\dagger} P<0.05$, versus IR group. There were significant differences of apoptosis index in P1, P2, P3 groups than that in IR group; ${ }^{\ddagger}$ versus $\mathrm{P} 1$ group. There were significant apoptosis in $\mathrm{P} 2$ and $\mathrm{P} 3$ groups. But, in $72 \mathrm{~h}$, there was no obvious difference between P2 and P3 group.

the nucleus and mitochondrion changes levels were also alleviated obviously (Figure 5).

3.5. Quantification of AQP4. Immunoblot analysis showed AQP4 protein in brain homogenates from rats as bands at about 32 and $34 \mathrm{kDa}$ representing the two AQP4 protein isoforms expressed in brain (Figure 6). Quantitative analysis of brain homogenates indicated increased AQP4 protein in the IR group and PCr group compared with those in the sham-operated group. The levels of brain AQP4 protein were decreased in the PCr group compared with the IR group $(P<0.01$, Figure 7$)$.

\section{Discussion}

Rapid decrease of ATP has been shown after cerebral ischemia, which leads to secondary injuries. We hypothesized that supplication of an exogenous substrate before ischemia reperfusion might alleviate IR damages. Study showed that [5, 10], after exogenous injection, the distribution of disodium creatine phosphate is mainly in skeletal muscle and myocardium, brain is the second one, and kidney is the third, but less in lung and liver. So the concentration of $\mathrm{PCr}$ is high

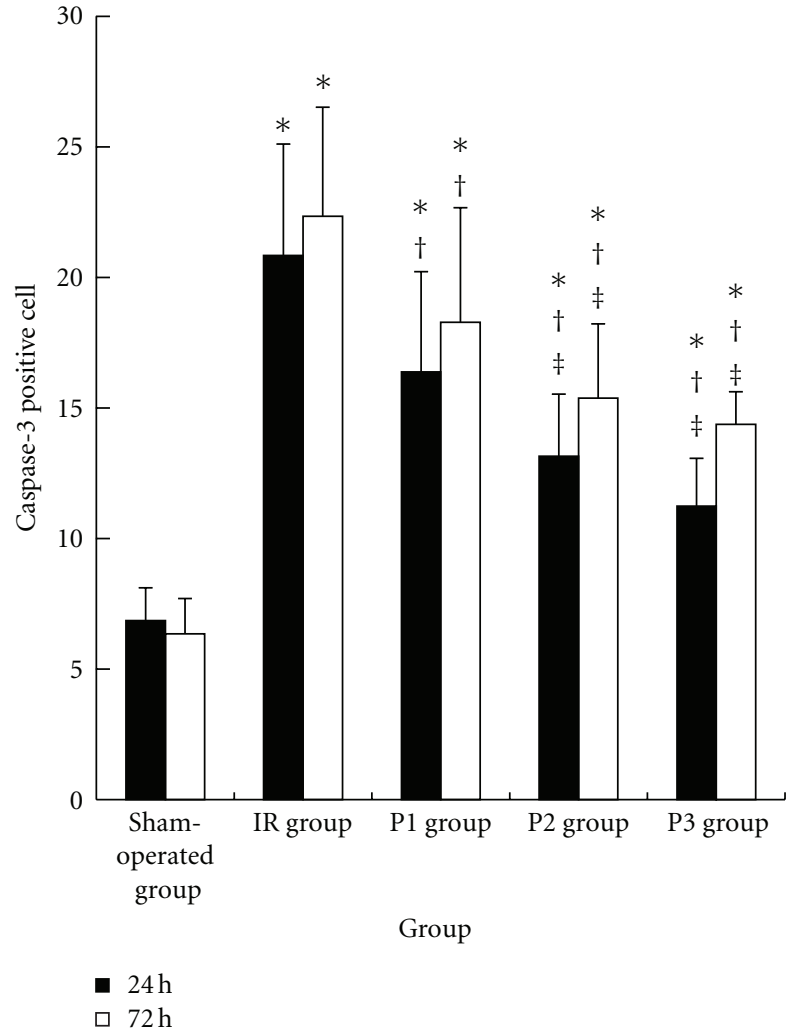

Figure 3: Caspase-3 positive cells in brain slices of five groups at $24 \mathrm{~h}$ and $72 \mathrm{~h}$. Data are presented as means $\pm \mathrm{SD}$. ${ }^{*} P<0.01$, versus sham-operated group. There were significant differences in IR, P1, $\mathrm{P} 2, \mathrm{P} 3$ groups than that in sham-operated group; ${ }^{\dagger} P<0.05$, versus IR group. There were significant differences in P1, P2, P3 groups than that in IR group; ${ }^{\ddagger}$ versus P1 group. There were significant apoptosis in P2, P3 groups. But, in $72 \mathrm{~h}$, there was no obvious difference between $\mathrm{P} 2$ and $\mathrm{P} 3$ group.

in brain after administration. Investigation showed that $\mathrm{PCr}$ can go through the blood-brain barrier, even through the cell membrane $[11,12]$, to supply energy to cells directly. But, in actual practice, we do not use disodium creatine phosphate to protect brain from damage at present, especially in early stage ( 3 hs after infarction) of cerebral ischemia. So the study was performed to investigate the potential effects of $\mathrm{PCr}$ on neurologic behavioral assessments, ultrastructural histopathologic changes, and neuronal apoptosis in an animal model of focal cerebral ischemia-reperfusion.

Studies have shown that the caspase family is the promoter and implementer of apoptosis in mammalian cells, among which, caspase- 3 is the most critical downstream apoptosis protease in the caspase cascade "waterfall" [12]. A variety of extracellular signals activate caspase- 8 through Fas receptor pathway and caspase-9 via mitochondrial cytochrome $\mathrm{C}$ in cerebral ischemia reperfusion injury. Activation of caspase- 8 and caspase- 9 then promote caspase-3, which in turn hydrolyzes cell-specific proteins, and polyADP ribose polymerase (PARP), thereby inducing apoptosis $[13,14]$.

Many investigators found the expression of caspase- 3 enhanced with the increased cell apoptosis in brain, both in 


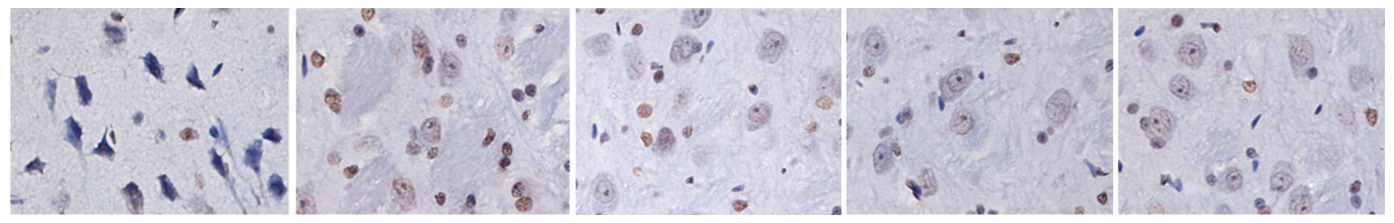

(a)

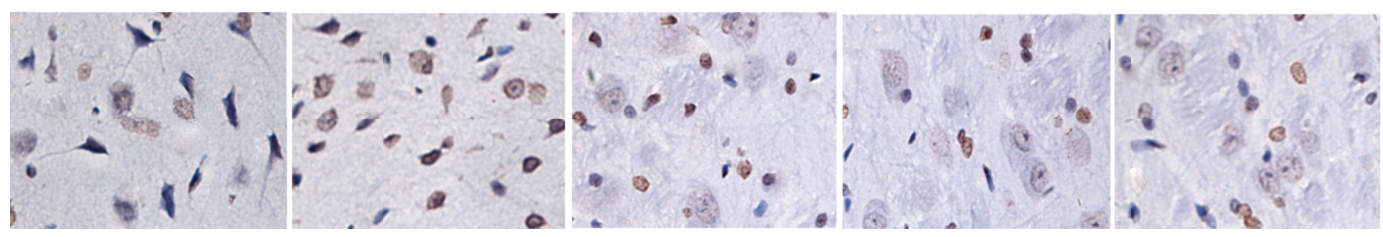

(b)
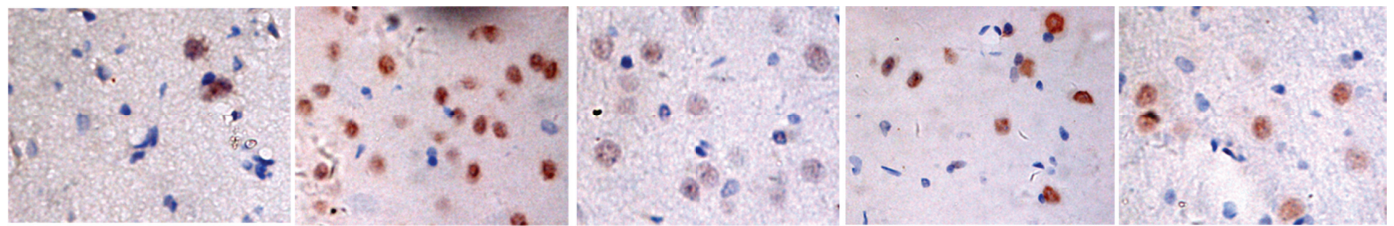

(c)

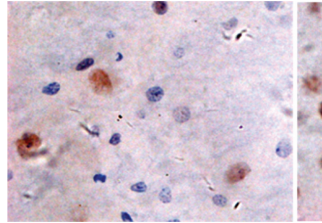

$S$ group

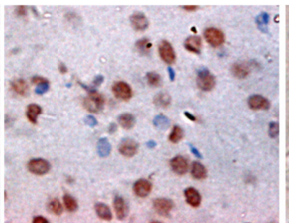

IR group

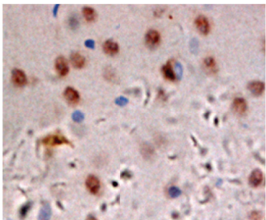

P1 group

(d)

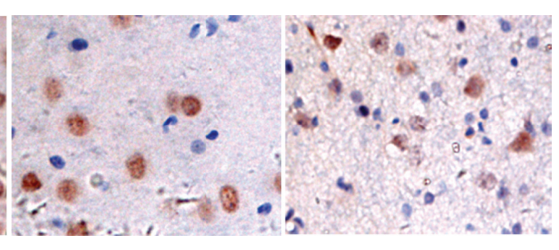

P2 group

P3 group

FIgURE 4: (a) Apoptosis cells in brains by TUNEL assay after $24 \mathrm{~h}$ ischemia $(\times 400)$. (b) Apoptosis cells in brains by TUNEL assay at $72 \mathrm{~h}$ ischemia $(\times 400)$. (c) Apoptosis cells in brains by caspase- 3 immunohistochemical stain assay after $24 \mathrm{~h}$ ischemia $(\times 400)$. (d) Apoptosis cells in brains by caspase- 3 immunohistochemical stain assay after $72 \mathrm{~h}$ ischemia $(\times 400)$.
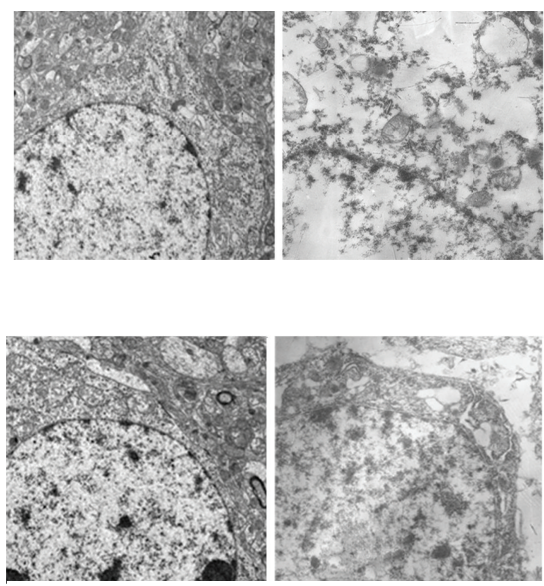

$S$ group

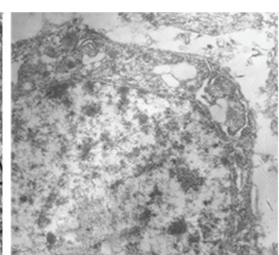

IR group
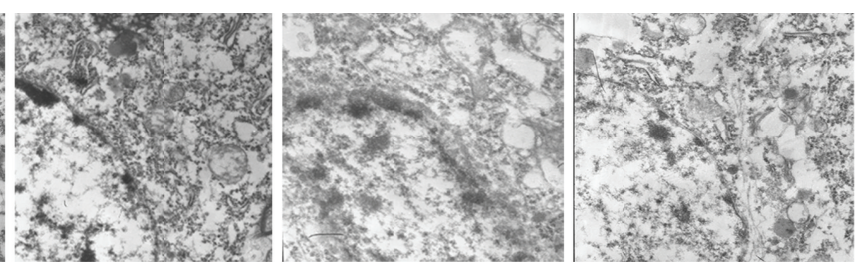

(a)

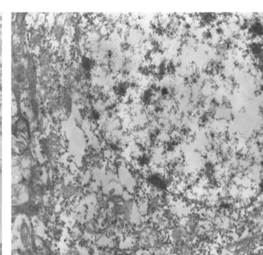

P1 group

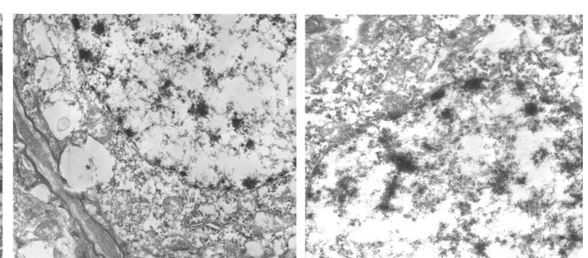

P2 group
P3 group

(b)

Figure 5: (a) Hippocampus ultrastructural histopathologic changes in five groups at $24 \mathrm{~h}(\times 5000)$. (b) Hippocampus ultrastructural histopathologic changes in five groups at $72 \mathrm{~h}(\times 5000)$. 


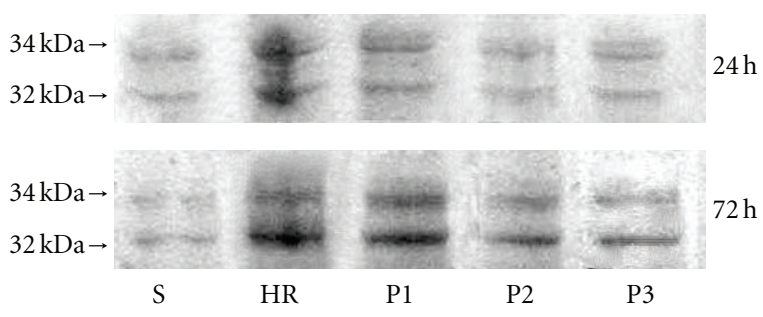

FIgURE 6: Western Blot analyses of AQP4. 32 and $34 \mathrm{kDa}$ representing the two AQP4 protein isoforms expressed in brain.

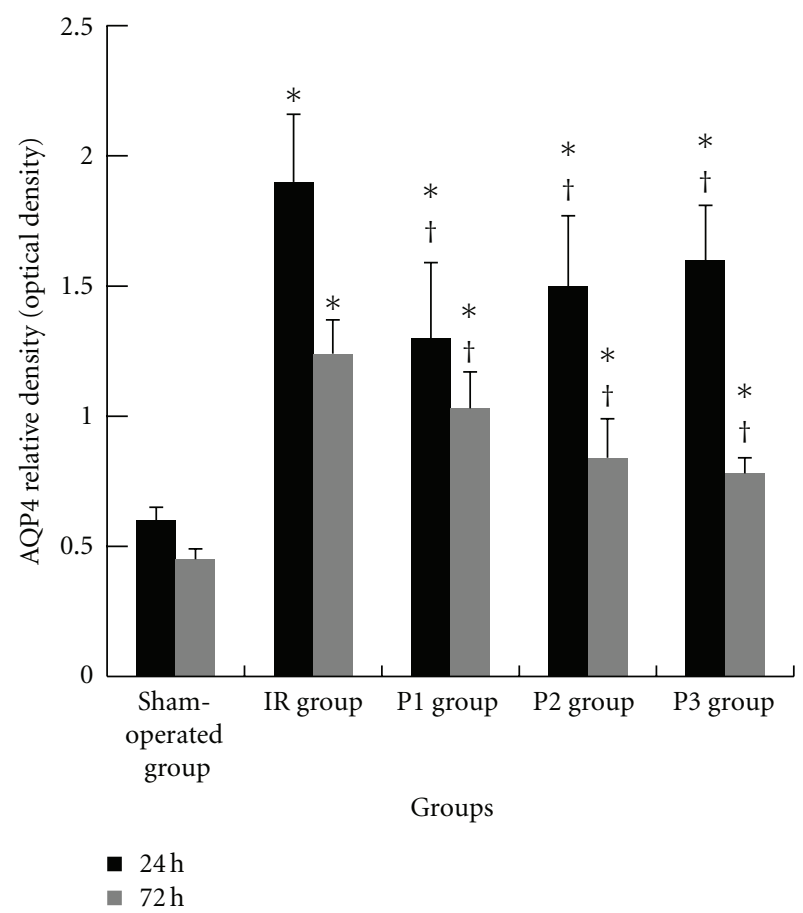

FIgURe 7: AQP4 relative density of five groups. ${ }^{*} P<0.01$, versus sham-operated group; ${ }^{\dagger} P<0.05$, versus IR group. There were significant differences in P1, P2, P3 groups than that in IR group. But there was no obvious differences between $\mathrm{P} 1, \mathrm{P} 2$, and $\mathrm{P} 3$ groups in AQP4 relative density.

local and focal ischemia. The number and region of apoptosis were related to ischemia time and sensitivity of neurons. After cerebral ischemia, apoptosis cells mainly located in selective infarction area, preoptic region, corpus striatum, inner margin cortex of infarction boundary, corpus striatum, hippocampus, and olfactory tubercle [15] peak in $24-48 \mathrm{~h}$. In the study, we examined cell apoptosis by TUNEL and caspase-3 immunohistochemical staining, and we observed obviously decrease of AI and caspase-3 positive cells after intervention of PCr. These data suggest that PCr reduce caspase-3 expression after cerebral IR injury.

Cerebral ischemia reperfusion leads to diverse structural changes in neural cells. Some brain regions, including the hippocampus, are more vulnerable to ischemia than others. In the experiment, we observed swelling mitochondrial, disaggregation of polyribosomes, decreased rough endoplasmic reticulum and Golgi apparatus in postischemic hippocampal neurons, which is consistent with the studies before [16]. Mitochondrial swelling is one of the initial postischemic changes. In mild cases, swollen mitochondria regain their normal shape soon. But, in severe cases, organelles in neurons demonstrate condensation, increased matrix density, and deposits of an electron-dense material followed by the disintegration of mitochondria $[17,18]$. In the experiment, we proved fewer mitochondria changes after intervention of PCr. And we also demonstrated slightly shrunken nucleus and complete nuclear membrane in the PCr pretreatment group compared with the significantly aggregated chromatin toward the nuclear membrane in the IR group.

Recently, the bidirectional water channel aquaporin-4 (AQP4) has been found to play an important role in brainwater homeostasis [19]. AQP4 protein is expressed strongly in astroglia at the BBB and CSF-brain interfaces [20], involved in water movement between fluid compartments (blood and CSF) and brain parenchyma. It has been suggested that AQP4 deletion markedly reduced brain swelling of cytotoxic brain edema, including water intoxication and focal cerebral ischemia $[21,22]$. Thus, AQP4 appears to facilitate water movement in cytotoxic edema. So detection of expression level of AQP4 can indirectly determine the brain swelling extent in cerebral ischemia. In the study, according to ultrastructure observation, less swelling can be seen in plasma and organelles; meanwhile, we detected significantly decrease of AQP4 expression, so PCr can alleviated brain swelling in cerebral IR injury, although PCr did not decrease ICP significantly.

In the study, three doses of $\mathrm{PCr}$ have been adopted to prove the effect of $\mathrm{PCr}$ on focal cerebral ischemia-reperfusion injury. After pretreatment in three groups, animals' IR injuries were alleviated in motor behavior, apoptosis, brain swelling, and ultrastructural pathological changes. The effects of $200 \mathrm{mg} / \mathrm{kg}$ and $400 \mathrm{mg} / \mathrm{kg}$ were not significantly different, but the side effect of PCr increased with higher dosage, especially low blood pressure. So, in the experiment, $200 \mathrm{mg} / \mathrm{kg}$ is more appropriate for treatment.

\section{Conclusions}

In conclusion, the study demonstrates PCr has neuroprotective effect on focal cerebral IR injury, which may relate to apoptosis. After PCr pretreatment, neurobehavioral score, infarction volume, and brain swelling are alleviated.

\section{Conflict of Interests}

The authors have declared that no conflict of interests exists.

\section{Acknowledgment}

The study is supported by a grant from the Science Technology Plan of Liaoning Province (No. 2011225038). 


\section{References}

[1] M. Konig, M. Klotz, L. Heuser et al., "Perfusion CT in acute stroke: characterization of cerebral ischemia using parameter images of cerebral blood flow and their therapeutic relevance clinical experience,” Electromedia, vol. 66, pp. 61-67, 1998.

[2] M. Wyss and R. Kaddurah-Daouk, "Creatine and creatinine metabolism," Physiological Reviews, vol. 80, no. 3, pp. 1107 1213, 2000.

[3] E. S. Rawson and A. C. Venezia, "Use of creatine in the elderly and evidence for effects on cognitive function in young and old," Amino Acids, vol. 40, no. 5, pp. 1349-1362, 2011.

[4] P. Luisa, C. Aroldo, L. L. Gian, C. Principato, C. Gandolfo, and M. Balestrino, "Kinetics of creatine in blood and brain after intraperitoneal injection in the rat," Brain Research, vol. 974, no. 1-2, pp. 37-42, 2003.

[5] M. Balestrino, M. Lensman, M. Parodi et al., "Role of creatine and phosphocreatine in neuronal protection from anoxic and ischemic damage," Amino Acids, vol. 23, no. 1-3, pp. 221-229, 2002.

[6] W. Zhiqiang, Z. Xiaoning, T. Jiang et al., "Relationship between neural score and infarction volume of rats in cerebral infarction," Clinical Neurology of China, vol. 16, no. 4, pp. 394399, 2008.

[7] E. Z. Longa, P. R. Weinstein, S. Carlson, and R. Cummins, "Reversible middle cerebral artery occlusion without craniectomy in rats," Stroke, vol. 20, no. 1, pp. 84-91, 1989.

[8] G. A. Rosenberg, S. Mun-Bryce, M. Wesley, and M. Kornfeld, "Collagenase-induced intracerebral hemorrhage in rats," Stroke, vol. 21, no. 5, pp. 801-807, 1990.

[9] M. C. Papadopoulos and A. S. Verkman, "Aquaporin-4 gene disruption in mice reduces brain swelling and mortality in pneumococcal meningitis," Journal of Biological Chemistry, vol. 280, no. 14, pp. 13906-13912, 2005.

[10] R. Wang, "Pharmocokinetics and clinical application of phosphocreatine," Capital Medicine, vol. 5, article 32, 1999.

[11] R. A. Pattillo, B. D. Collier, H. Abdel-Dayem et al., "Phosphorus-32-chromic phosphate for ovarian cancer: I. Fractionated low-dose intraperitoneal treatments in conjunction with platinum analog chemotherapy," Journal of Nuclear Medicine, vol. 36, no. 1, pp. 29-36, 1995.

[12] G. Prabhakar, L. Vona-Davis, D. Murray, P. Lakhani, and G. Murray, "Phosphocreatine restores high-energy phosphates in ischemic myocardium: Implication for off-pump cardiac revascularization," Journal of the American College of Surgeons, vol. 197, no. 5, pp. 786-791, 2003.

[13] B. B. Cho and L. H. Toledo-Pereyra, "Caspase-independent programmed cell death following ischemic stroke," Journal of Investigative Surgery, vol. 21, no. 3, pp. 141-147, 2008.

[14] B. R. Broughton, D. C. Reutens, and C. G. Sobey, "Apoptotic mechanisms after cerebral ischemia," Stroke, vol. 40, no. 5, pp. 331-339, 2009.

[15] F. Moroni, "Poly(ADP-ribose)polymerase 1 (PARP-1) and postischemic brain damage," Current Opinion in Pharmacology, vol. 8, no. 1, pp. 96-103, 2008.

[16] Y. Li, M. Choop, N. Jiang et al., "Cell death induced bu middle to sereve focal cerebral ischemia (10-120 minutes) associated with internucleosomal DNA cleavage in the rat," Stroke, vol. 26, article 182, 1995.

[17] C. K. Petito and W. A. Pulsinelli, "Delayed neuronal recovery and neuronal death in rat hippocampus following severe cerebral ischemia: possible relationship to abnormalities in neuronal processes," Journal of Cerebral Blood Flow and Metabolism, vol. 4, no. 2, pp. 194-205, 1984.
[18] N. J. Solenski, C. G. DiPierro, P. A. Trimmer, A. L. Kwan, and G. A. Helms, "Ultrastructural changes of neuronal mitochondria after transient and permanent cerebral ischemia," Stroke, vol. 33, no. 3, pp. 816-824, 2002.

[19] T. Kovalenko, I. Osadchenko, A. Nikonenko et al., "Ischemiainduced modifications in hippocampal CA1 stratum radiatum excitatory synapses," Hippocampus, vol. 16, no. 10, pp. 814825, 2006.

[20] M. Amiry-Moghaddam, T. Otsuka, P. D. Hurn et al., "An $\alpha$-syntrophin-dependent pool of AQP4 in astroglial end-feet confers bidirectional water flow between blood and brain," Proceedings of the National Academy of Sciences of the United States of America, vol. 100, no. 4, pp. 2106-2111, 2003.

[21] J. E. Rash, T. Yasumura, C. S. Hudson, P. Agre, and S. Nielsen, "Direct immunogold labeling of aquaporin-4 in square arrays of astrocyte and ependymocyte plasma membranes in rat brain and spinal cord," Proceedings of the National Academy of Sciences of the United States of America, vol. 95, no. 20, pp. 11981-11986, 1998.

[22] G. T. Manley, M. Fujimura, T. Ma et al., "Aquaporin-4 deletion in mice reduces brain edema after acute water intoxication and ischemic stroke," Nature Medicine, vol. 6, no. 2, pp. 159-163, 2000 . 

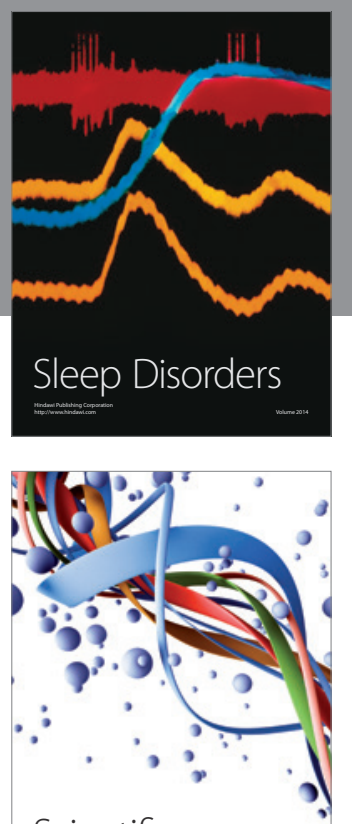

Scientifica
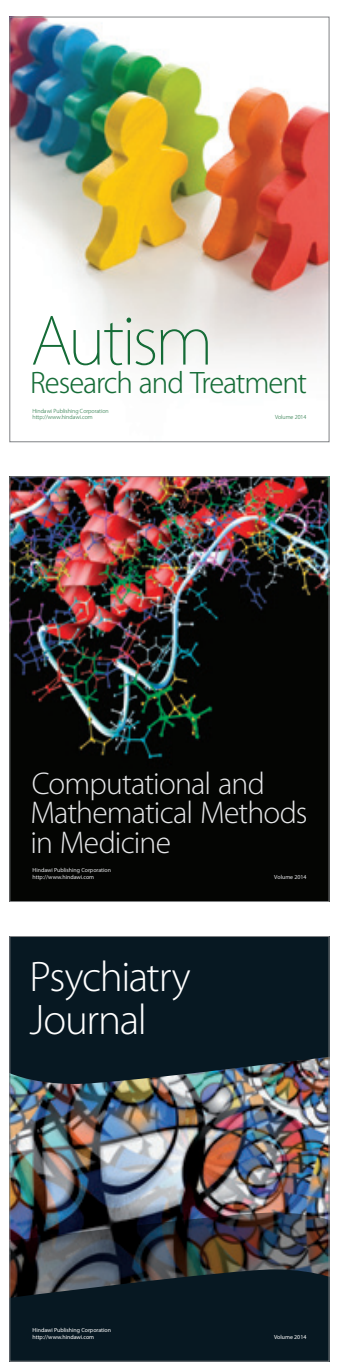
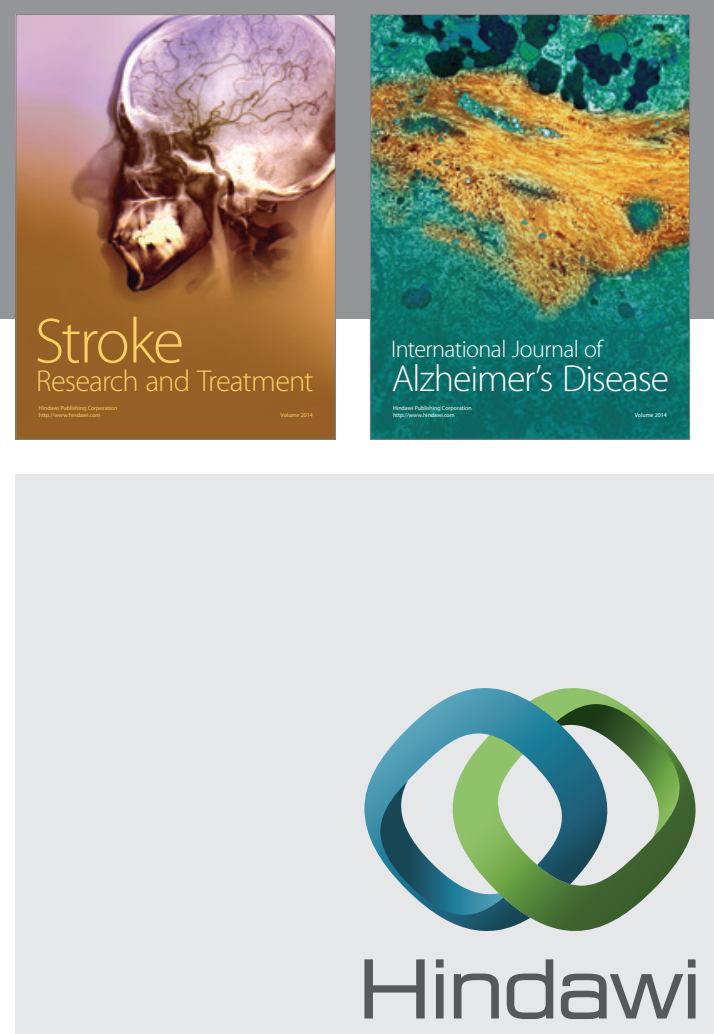

Submit your manuscripts at

http://www.hindawi.com
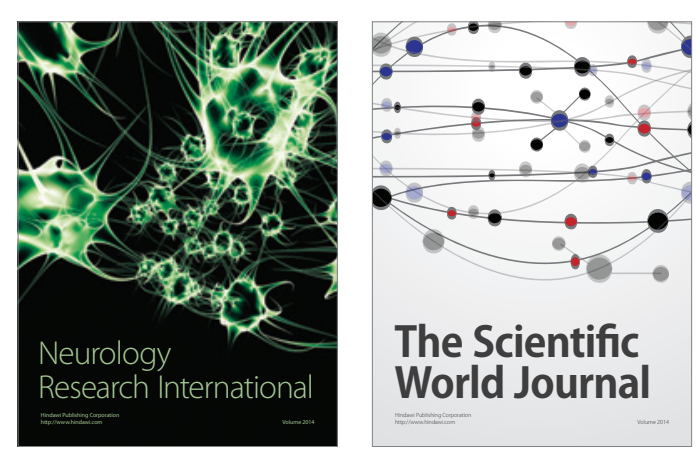

The Scientific World Journal

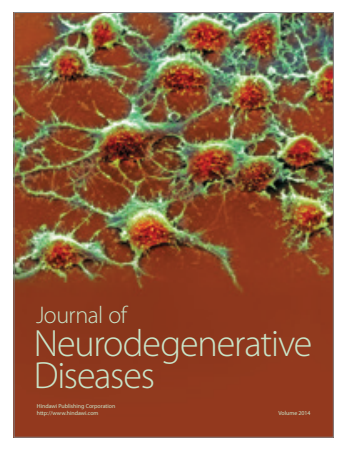

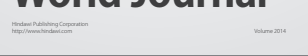

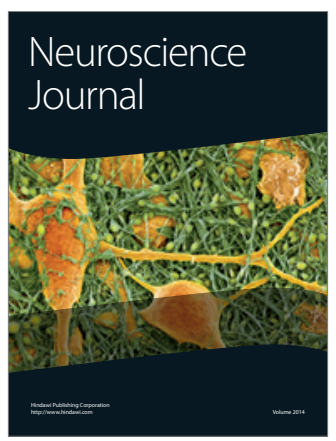

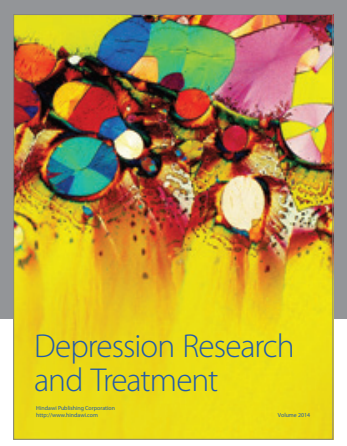
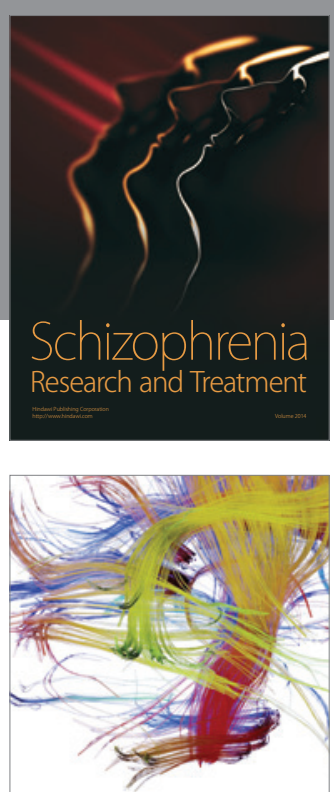

Brain Science

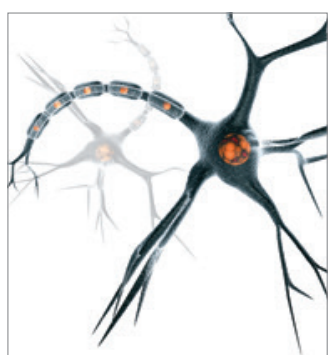

Neural Plasticity
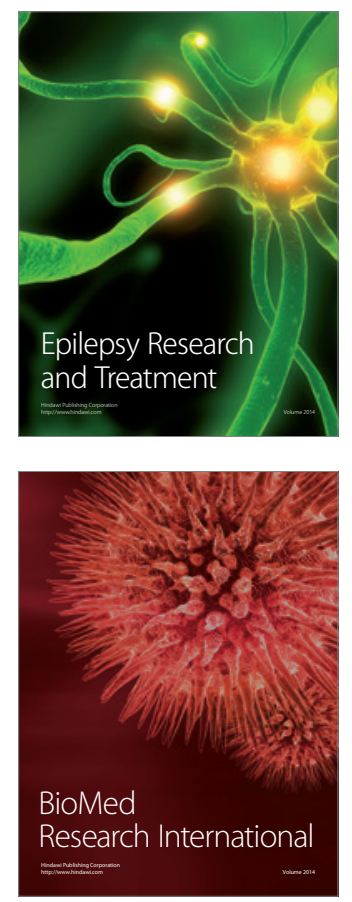

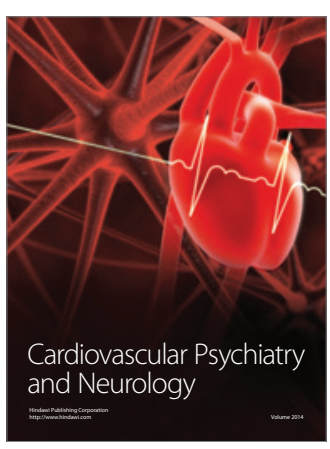

Parkinson's

Disease
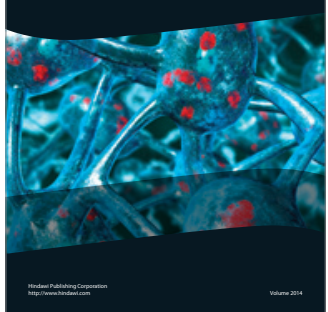